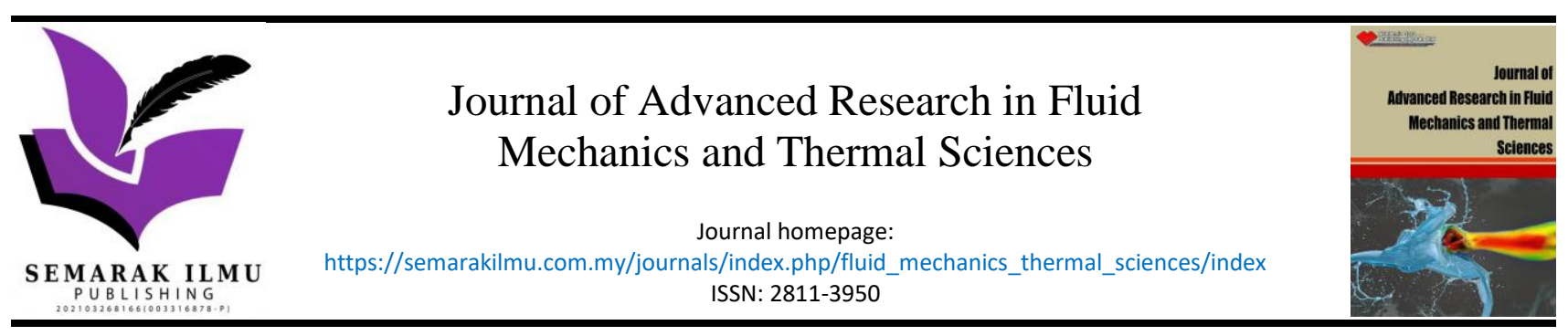

\title{
Improving Electromechanical Impedance Damage Detection Under Varying Temperature
}

\author{
Mohamed Djemana ${ }^{1}$, Meftah Hrairi ${ }^{2,}{ }^{*}$, Norfazrina Hayati Mohd Yatim ${ }^{2}$ \\ Second Cycle Department, Higher School of Industrial Technologies, P.O. Box .218, 23000, Annaba, Algeria \\ 2 Department of Mechanical Engineering, Faculty of Engineering, International Islamic University Malaysia, PO Box 10, 50728 Kuala Lumpur, \\ Malaysia
}

\section{ARTICLE INFO}

\section{Article history:}

Received 14 August 2021

Received in revised form 25 December 2021

Accepted 12 January 2022

Available online 3 February 2022

\section{ABSTRACT}

\begin{abstract}
The field of structural health monitoring has seen a fundamental shift in recent years, as researchers strive to replace conventional non-destructive evaluation techniques with smart material-based techniques. Perhaps the most promising of smart material techniques for developing structural health monitoring (SHM) systems is electromechanical impedance (EMI) which can be used for real-time structural damage assessment. In EMI, mechanical resonances of structure can be seen in electrical characteristics of piezoelectric transducers due to electromechanical coupling of transducer with the structure. Existence of damage will cause a structural stiffness change and therefore the resonant characteristics of the structure will be altered. This article presents an experimental and numerical study to investigate the effects of notch damage with temperature on the electrical impedance of the piezoelectric sensor used in the EMI technique. The practical implementation of the compact EMI method utilizes as its main apparatus an impedance analyser (Model Agilent 4294A) that reads the insitu EMI of piezoelectric wafer active sensors (PWAS) attached to the monitored structure. The finite element modelling used ANSYS software three-dimensional (3D) capability to simulate an aluminium beam at varying temperatures. Real-time monitoring of the structure is achieved based on harmonic measurements. The results conclusively showed that the proposed temperature compensation technique eliminates the results ambiguity and enabled the EMI system to detect small damages that were otherwise indiscernible.
\end{abstract}

\section{Introduction}

Maintaining the safety of a structure can be assured by means of Structural health monitoring (SHM). The assessment of a structural health is particularly important for aged aerospace vehicles and civil engineering structures subjected to heavy periodic loads. For such structures, SHM is a complex activity that involves the interaction of numerous factors. It has been shown that aerospace maintenance and repairs represent about a quarter of commercial fleet's operating costs [1]. One of the possible solutions to decrease these costs is to couple the selective use of condition-based

\footnotetext{
* Corresponding author.

E-mail address: meftah@iium.edu.my

https://doi.org/10.37934/arfmts.92.1.123133
} 
maintenance with the implementation of innovative SHM systems, which will continuously assess the structural integrity through online structural health monitoring. Continuous SHM would lead to considerable life-cycle cost reduction since the damaged part can be replaced in time, preventing the failure of the whole structure and the loss of human lives. Another critical feature of a SHM system is its ability to detect the initiation and growth of structural damage at a nascent stage. This would bring a considerable safety enhancement. Such safety enhancement creates a compelling reason to have high-quality online structural health monitoring (SHM) in both modern and aging structures [2].

Focus of intensive study for decades, the electromechanical impedance (EMI) method has demonstrated in several studies that it can be useful for complex and lab-scale structures. There are, however, several practical difficulties that have impeded the application of EMI to real-world structures in a useful and reliable way. Temperatures effects have lately been addressed in the scientific experiments as challenging of many practical models. Sepehry et al., [3] presented theoretical and experimental results by ISHM method in order to validate the proposed model to consider the temperature dependency of PWAS material properties, a temperature-dependent model is developed for a PWAS bonded to a Euler Bernoulli cantilever beam. The comparison of theoretical and experimental results demonstrates a good improvement in ISHM modelling where temperature variation is present. Baptista et al., [4] proposed an EMI system capable of real-time data acquisition from multiple sensors that incorporated a temperature compensation functionality, eliminating one cause of incorrect diagnoses in structural monitoring. The experimental results show conclusively that the proposed methodology is efficient and feasible for real-time SHM. Baptista et al., [5] Studied experimentally the effect of temperature on the electrical impedance of the 5H PZT sensors commonly used in the EMI technique. They found that the temperature effects were strongly frequency-dependent, which may motivate future research in the SHM field. Safaeifar and Karimi [6] looked at how temperature changes affected the natural frequencies of a monitored structure. It has been discovered that as the temperature rises, the inherent resonant frequency of a structure reduces. Wandowski et al., [7] suggested an approach for compensation of temperature influence on damage detection in Carbon fiber-reinforced polymer using electromechanical impedance method. Gianesini et al., [8] established a way to adjust for the temperature effect in the electromechanical impedance technique. The method is particularly general since it may be applied to nonlinear (polynomial) temperature and/or frequency dependences observed on the impedance signatures horizontal and vertical shifts. The compensation is performed via a computer program that may be easily integrated into real-time damage detection systems. In the temperature range of $24 \mathrm{C}$ to $80 \mathrm{C}$ and the frequency range of 10 to $90 \mathrm{kHz}$, this compensation technique was successfully applied to two aluminum beams and one steel pipe, limiting the effect of temperature changes on damage detection structural health monitoring systems. Abbas et al., [9] developed an experimental setup using an EMI technique to detect the presence of fatigue cracks in stainless steel (304) beams and assess the effect of temperature fluctuations on the electrical impedance of piezoelectric sensors. They conducted a series of experiments in a controlled temperature setting $\left(25^{\circ} \mathrm{C}-160^{\circ} \mathrm{C}\right)$. The dielectric constant $\varepsilon_{33}^{T}$ which is known as the temperature-dependent constant of the PZT sensor, has been found to have a significant impact on the electrical impedance signature. Furthermore, the effective frequency shift (EFS) approach was used to provide significant temperature compensation for the current impedance signature of the PZT sensor in comparison to the reference signature over the measurement system's extended frequency bandwidth.

It is known that temperature and stress are two important environmental loads in practical engineering fields, and the correlated EMI research referring to temperature and stress load have also been reported [10-13]. In this paper, the impact of varying temperatures on sensor impedance signatures is experimentally and numerically studied. It is to be noted that, most of the existing works 
have considered this problem within the context of experimental set up or otherwise mathematical modelling but none have taken into consideration the effect of temperature with notch damage. This study was carried out experimentally and numerically. In addition, compensation technique presented and discussed for the effects of temperature variation were accomplished through a variety of methods. Therefore, the results presented here are of unique significance and novelty.

\section{Electromechanical Impedance Technique}

\subsection{Theoretical Background}

The EMI method for SHM and Non-Destructive Evaluation (NDE) is based upon changes in structural impedance at its drive point that's mean point of concentration or point at which the structural behavior/properties may change. These changes identify damage in the structure. The change is sensed electrically through changes in the apparent EMI of the piezoelectric transducer that is coupled to the host structure and is defined by

$$
Z(\omega)=\left[i \omega C\left(1-k_{31}^{2}\left(\frac{z_{s t r}(\omega)}{Z_{P Z T}(\omega)+Z_{s t r}(\omega)}\right)\right)\right]^{-1}
$$

where $Z(\omega)$ is the equivalent electro-mechanical admittance as seen at the PZT transducer terminals, $\kappa_{31}$ is the electro-mechanical cross coupling coefficient of the PZT transducer $\left(\kappa_{31}=d_{13} / \sqrt{\bar{s}_{11} \bar{\varepsilon}_{33}}\right), C$ is the zero-load capacity of the PZT transducer, $Z_{s t r}$ is the impedance of the structure, and $Z_{P Z T}$ is the impedance of the PZT transducer [14]. The piezoelectric sensor-actuators (wafer transducers) are closely bonded to the structure, and their EMI variation is measured across a wide frequency spectrum located in the high $\mathrm{kHz}$ band of frequencies. The transducer frequency response, phase, and amplitude, the impedance response (both real and imaginary), and other significant physical parameters act as indicators of potential structure damage and reflect the level of structural integrity. The transducer approach has proven most effective in the ultrasonic range of frequencies, where changes in local dynamics due to the initiation of damage within the structure are captured. Changes at this nascent stage are very small and have minimal effect on the global dynamics of the structure, making them difficult to detect with traditional low frequency vibration techniques [15].

\subsection{Damage Sensitive Indexes}

Basic structural damage is typically characterized with the use of damage indices. Two of the more commonly used indices include the root mean square deviation (RMSD) and the correlation coefficient deviation metric (CCDM). When a structure is considered healthy, baseline, or reference, EMI signatures are taken. These are compared to later signatures to assess structure health.

The Euclidean norm is the basis for the RMSD index; several variations of this index can be found in the literature. In this study, the RMSD index was calculated by

$$
R M S D=\sqrt{\frac{\sum_{k=1}^{N}\left(R_{E}\left(Z_{E, D}(k)\right)-R_{E}\left(Z_{E, H}(k)\right)\right)^{2}}{\sum_{k=1}^{N}\left(R_{E}\left(Z_{E, H}(k)\right)\right)^{2}}}
$$

where the subscripts $D$ and $H$ indicate damaged and healthy conditions, respectively; $R_{E}\left(Z_{E, D}(k)\right)$ and $R_{E}\left(Z_{E, H}(k)\right)$ are the real parts of the electrical impedance signatures acquired by the measurement 
system of the structure's damaged and healthy states, respectively. The range of frequency $k$ is measured between the initial and final frequencies. We use the real part of the impedance because it is known to be more reactive to damage or changes in the structure's integrity, and less sensitive to ambient temperature changes compared to the imaginary part.

The correlation coefficient is the basis for the second index, CCDM, and is calculated by

$C C D M=1-C_{C}$

Where $C_{C}$ is the correlation coefficient calculated using the real part of the electrical impedance signatures for the structure under healthy and damaged conditions, as defined before, in the same frequency range. It is calculated using the following equation [16].

$C_{C}=\frac{\sum_{k=1}^{N}\left(R_{E}\left(Z_{E, H}(k)\right)-\overline{R_{E}\left(Z_{E, H}\right)}\right)\left(R_{E}\left(Z_{E, D}(k)\right)-\overline{R_{E}\left(Z_{E, D}\right)}\right)}{\sqrt{\sum_{k=1}^{N}\left(R_{E}\left(Z_{E, H}(k)\right)-\overline{R_{E}\left(Z_{E, H}\right)}\right)^{2}} \sqrt{\sum_{k=1}^{N}\left(R_{E}\left(Z_{E, D}(k)\right)-\overline{R_{E}\left(Z_{E D}\right)}\right)^{2}}}$

\section{Methodology}

\subsection{Experimental Setup}

The experimental set-up consists of an impedance analyzer (Model Agilent 4294A), a personal computer equipped with data acquisition software, and an interface cable, as shown in Figure 1. Several experiments were performed using an aluminum alloy 1100 structure with dimensions of 500 $\mathrm{mm} \times 30 \mathrm{~mm} \times 1 \mathrm{~mm}$ and a mass of $4.12 \mathrm{~g}$. A PIC151 PZT patch with dimensions of $10 \mathrm{~mm} \times 10 \mathrm{~mm}$ $\times 1 \mathrm{~mm}$ was bonded $20 \mathrm{~mm}$ from the end of the specimen. The beam was heated with a temperature controller and a thermocouple to measure the temperature of the structure. Simultaneously, the impedance analyzer was used to test the electric impedance spectra of the sensor. Experiments were performed at temperatures between 27 and $50^{\circ} \mathrm{C}$ in steps of $5^{\circ} \mathrm{C}$. According to the location of piezoelectric resonance peaks appeared in the impedance spectra, the frequency range of 18.5-20.5 $\mathrm{kHz}$.

An uncertainty analysis was performed to evaluate the accuracy of the data in this study. This analysis was conducted on the temperature which is the only measured quantity. The total uncertainty, $u_{\text {total, }}$, for the thermocouple is estimated according to the procedure reported in the literature, and this is given by the relation [17]

$u_{\text {total }}=\sqrt{u_{\text {inst }}^{2}+u_{\text {rand }}^{2}}$

where $u_{\text {inst }}$ is the instrument uncertainty based on the resolution and the accuracy of the temperature measurement caused by the voltage measurement and $u_{\text {rand }}$ is the random uncertainty based on the data fluctuation. Following this procedure, the uncertainty in the temperature was evaluated to be around $\pm 0.25^{\circ} \mathrm{C}$ which is primarily due to the instrument uncertainty. 


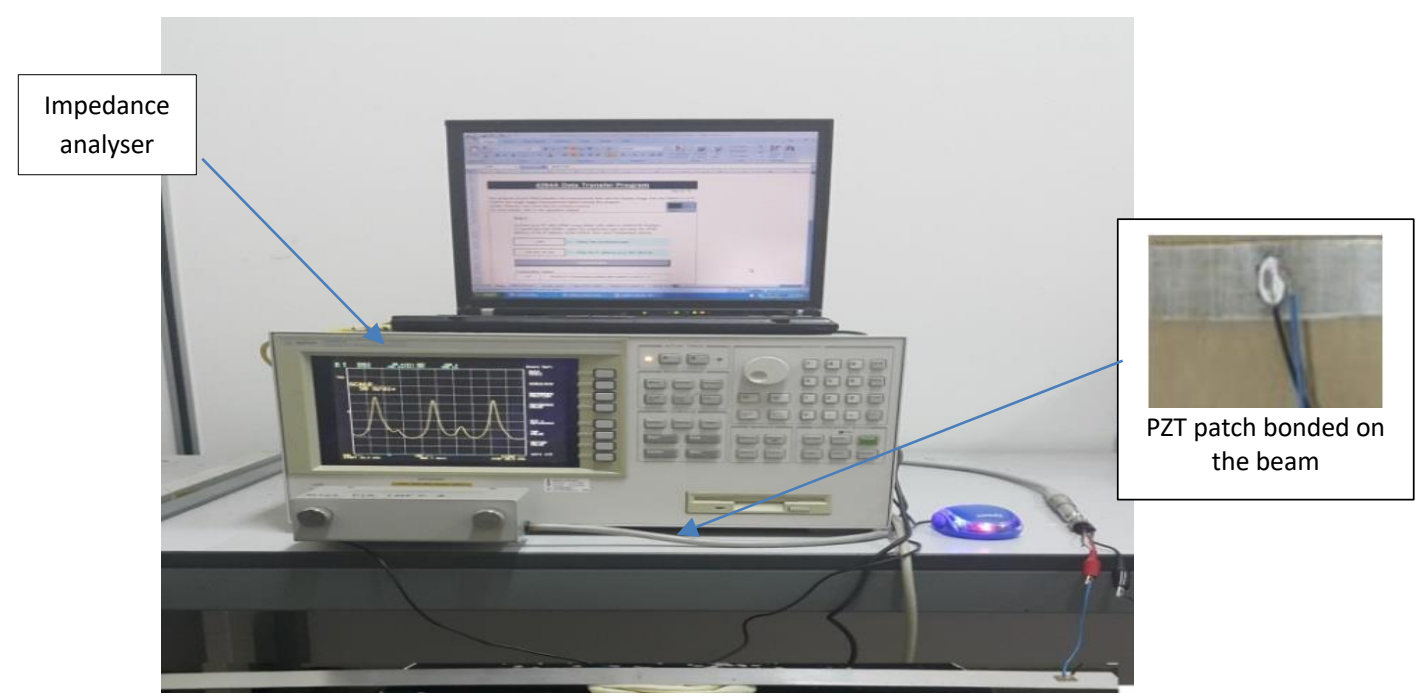

Fig. 1. Experimental setup

\subsection{Finite Element Modelling}

In this section, FE analysis is performed deterministically. The FE modelling was carried out in ANSYS software where the three dimensional (3D) 20-node parabolic SOLID226 element was used to build the structure and the PZT models. SOLID226 is defined as a coupled-field element with thermoelectric, piezoresistive and piezoelectric capabilities. These properties make SOLID226 an excellent choice to use with the complex problems inherent to our model. Notably, it includes the same full EM coupling that is seen in piezoelectric materials. Figure 2 shows the FE model of the PZT patch bonded on the healthy beam.

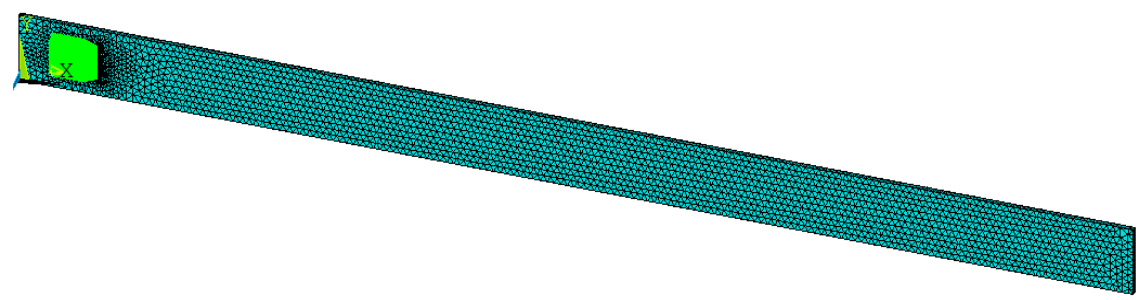

Fig. 2. Finite element model of beam structure

To be more realistic, the bonding layer (measured $0.03 \mathrm{~mm}$ ) was also simulated. A voltage of $1 \mathrm{~V}$ was applied on the top master node, whereas OV was applied on the bottom one (representing the ground contact). Multiphysics harmonic analyses have been performed with elaborated finite element model to generate the frequency plots of electromechanical impedance.

Our simulation was taken a step further with the inclusion of a temperature effect to the FEM dynamic simulation. It is known that temperature variation can not only change certain host structure properties but can also affect the PZT itself and its bonding layer [18]. The complete EMI system which is PZT bonded to the structure with temperature is simulated. 


\section{Results}

\subsection{Validation of the FE model}

To assess the accuracy of the proposed finite element model, it was subjected to a validation test benchmarked from the experiments. Validation is accepted based on approximation of curve trend, magnitude close reproduction of the experimental results. Figure 3 shows the comparison between the obtained simulation results and experimental results.

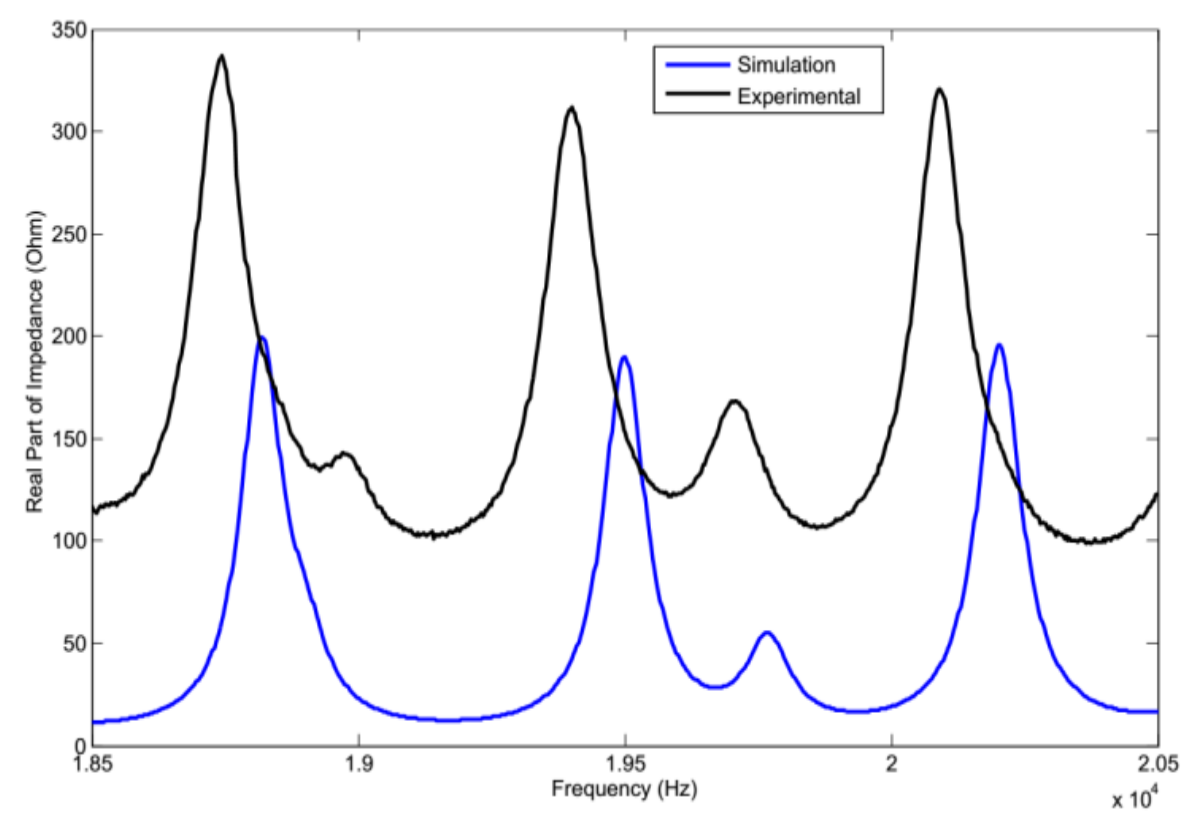

Fig. 3. Comparison between simulation and experimental results for an aluminium beam

\subsection{Temperature Effects on EMI}

Figure 4 shows the real part of the electromechanical impedance signatures for a healthy beam heated at temperatures between 27 and $50^{\circ} \mathrm{C}$ for frequencies from 18.5 to $21 \mathrm{kHz}$. As can be seen in Figure 4, there is a frequency shift to the left for the electromechanical signature due to the increase in temperature due the dielectric constant $\varepsilon_{33}^{T}$ which is recognized as the temperature-dependent constant of PZT sensor has sufficiently influenced the electrical impedance signature [9]. Similarly, as the temperature decreases, the signature shifts to the right. Variations in the electrical resistance and some vertical shifts are also observed. 


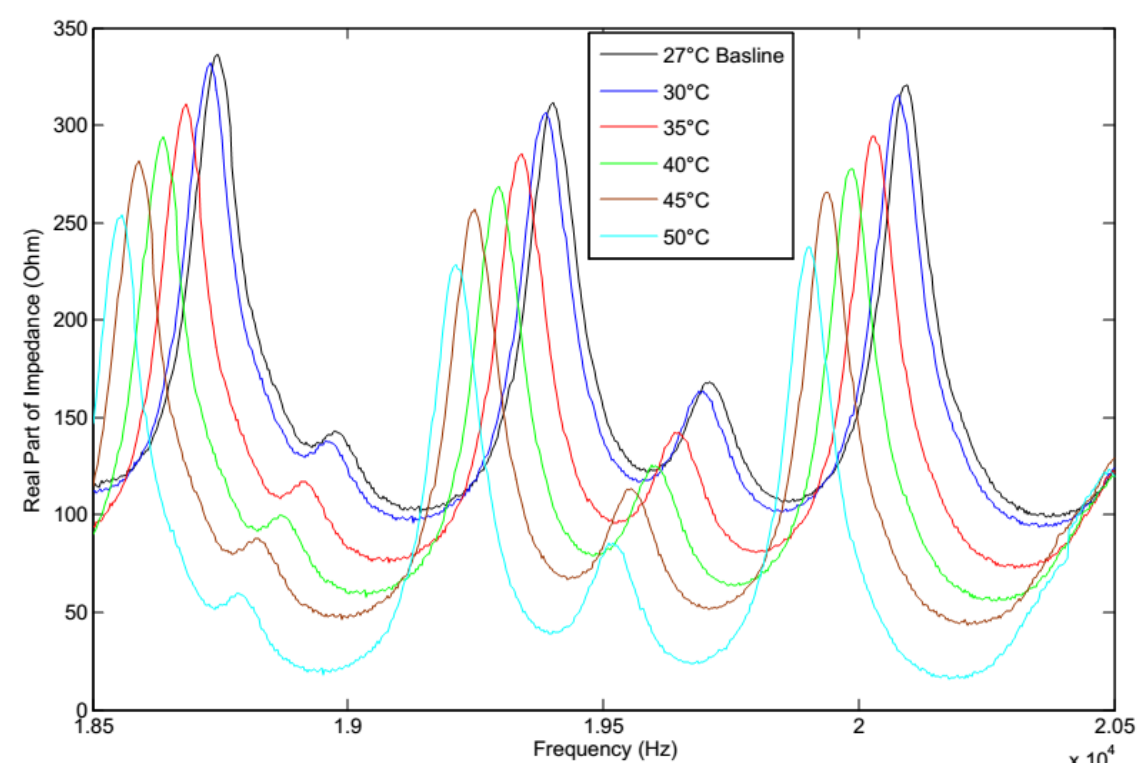

Fig. 4. Real part of the electromechanical impedances resulting from temperature changes

\subsection{Co-existence of Damage and Temperature}

Upon the inspection of the effect of temperature on EMI, we proceed to the effect of other types of damages, which are damage and temperature. Combining crack and temperature damage at the same time were also performed in this part. This attempt is expected to allow the researcher to model more realistic cases and to show that EMI systems can discover these problems. Figure 5 shows the differences between signals generated by the temperature that exists on the notched beam and the healthy beam. It can be seen that the electromechanical signature of the temperature that exist on the notched beam decrease compared with temperature signature and shifted to the left side. The decrease of the resonant peaks frequencies, which reflect the decreasing of local stiffness, is caused by the existence of local damage (notch). Therefore, the amount of frequency shift increases with the increasing of the notch depth [19].

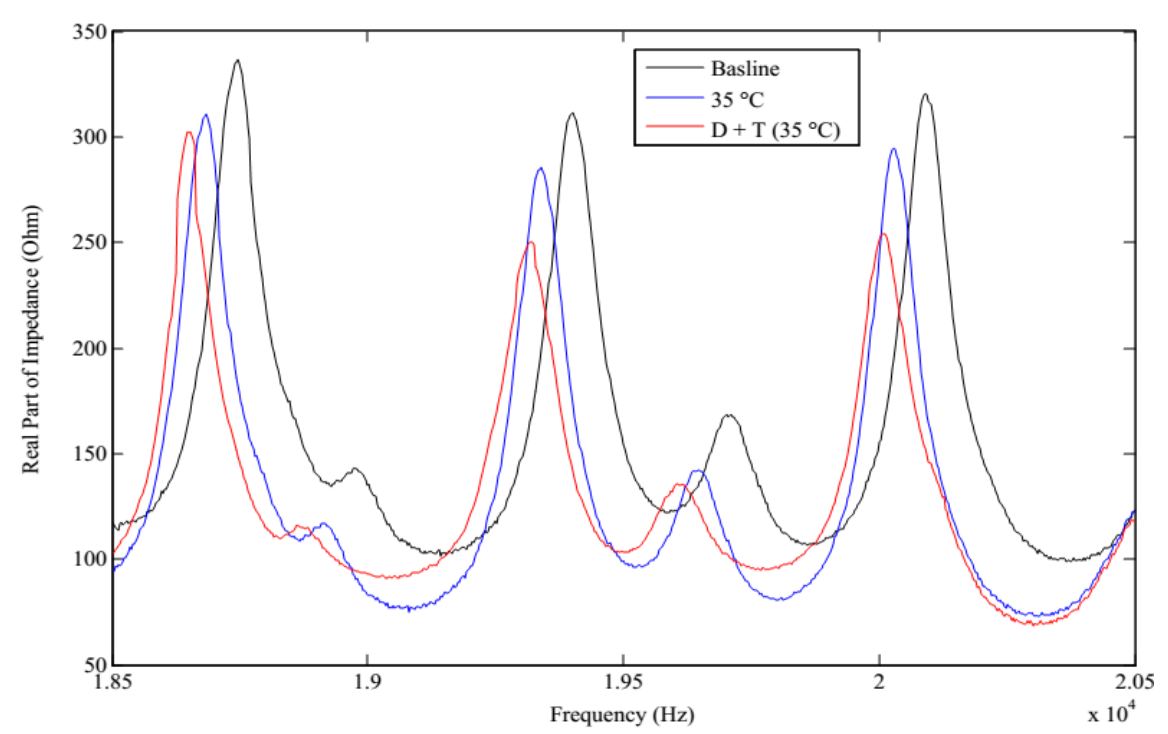

Fig. 5. Real part of the electromechanical impedance resulting from temperature changes 


\subsection{Compensation for Temperature Effects}

One major reason that has kept the EMI technique from being implemented in real world applications is that temperature variations are responsible for significant changes in the EMI signature. As a result, healthy monitored structures can be falsely diagnosed with damage due to a simple change in the surrounding temperature. Implementing real-time EMI monitoring will depend on methods that can automatically neutralize or reduce temperature effects. The model presented here is focused on the real component of the EMI signature since it is more sensitive to structural damage and less susceptible to temperature variation.

Compensation for the effects of temperature variation can be accomplished through a variety of methods. Park et al., [20] modified the RMSD index to compensate for shifts in both frequency and amplitude. Koo et al., [21] improved on the Park et al., [20] method by creating an effective frequency shift (EFS) that countered temperature variations. Sun et al., [22] compensated for the variations in signatures with a cross-correlation concept. Our approach is similar to Sun et al., [22] where similarities in two signals, the updated signatures and the baseline, are revealed to compensate for the frequency shifts. The cross correlation $R_{x y}$ of two signals $x(t)$ and $y(t)$ are defined by.

$R_{x y}(\tau)=\lim _{T \rightarrow \infty} \frac{1}{T} \int_{0}^{T} f_{x}(t) f_{y}(t+\tau) d t$

where $f_{x}(t)$ is the magnitude of the signal at point $x$, at time $t$, and $f_{y}(t+\tau)$ is the magnitude of the signal at a point $y$ at time $(t+\tau), \tau$ is the number of data points delayed. By varying, the relationship between the signals at $x$ and $y$ as a function of time is obtained. The following definition applies for sampled signals:

$R_{x y}(m)=\frac{1}{N} \sum_{n=1}^{N-m+1} x(n) y(n+m-1)$

where $m=1,2,3, \ldots, N+1$. The cross-correlation coefficient is

$\rho_{x y}(\tau)=\frac{R_{x y}(\tau)}{\sqrt{R_{x}(0) R_{y}(0)}}$

where $R_{x}(0)$ and $R_{y}(0)$ are the auto correlation functions of $\mathrm{x}$ and $\mathrm{y}$.

MATLAB was the software used to compensate the temperature effects. A MATLAB function is used to implement cross correlation function that obtains the baseline impedance signature of sensor, which is then used as reference to counter temperature variation effects. Additionally, the impedance of the PZT transducer was simulated for a healthy beam condition at a regulated temperature of $35^{\circ} \mathrm{C}$ (Figure 6).

Figure 6 demonstrates the compensation method result. Using Figure 3 as a reference, it appears that the compensation technique was successful in keeping temperature effects to a minimum. Since the purpose of the method was to eliminate false positive damage detection in healthy structures. The structure's impedance reconducted without temperature variation in order to assess structural damage. A compensation technique shows in different cases obtained over the previously stated range of 27 to $50^{\circ} \mathrm{C}$ with the step size of $18.6-21 \mathrm{kHz}$ and a baseline acquired at $27^{\circ} \mathrm{C}$. The results help identify the frequency shifts that occur with temperature variation. The shift profiles obtained can be used when evaluating the RMSD and CCDM indices in order to separate genuine damage detection from the temperature effects using the correlation coefficient Eq. (3). 


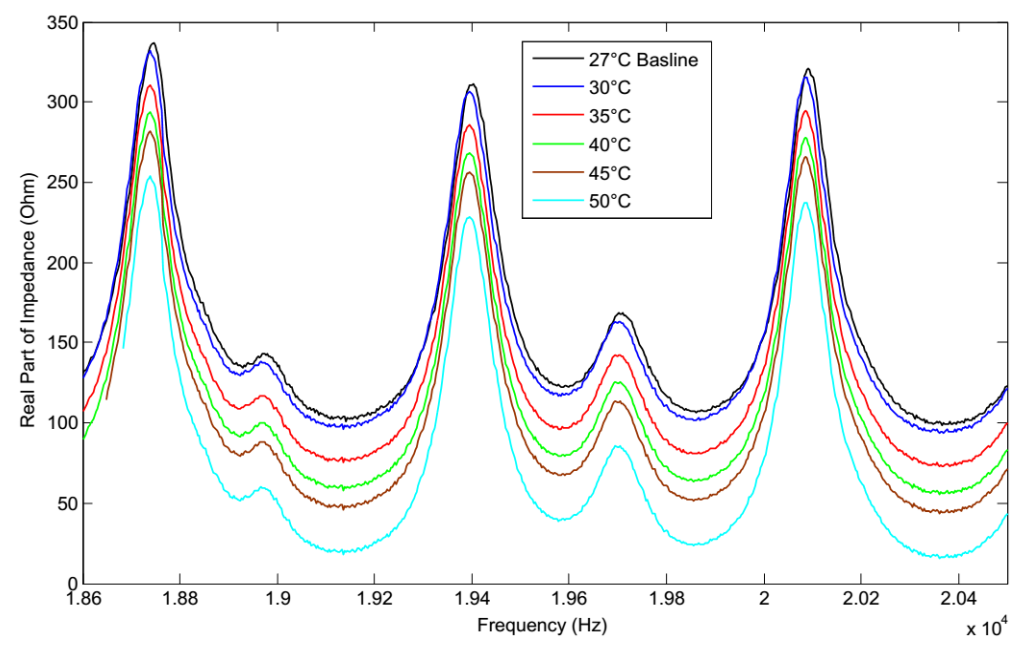

Fig. 6. Experimental results for real part of impedance signatures with the proposed compensation method for the temperature effects

To this end, the CCDM index is selected because it is insensitive to variations in the electrical impedance amplitude and responds instead to the shape of the impedance signature. This insensitivity makes the CCDM index ideal when considering the EMI variations due to temperature change. The part (a) from Figure 7 demonstrates that the signatures for damaged and healthy specimens at $35^{\circ} \mathrm{C}$ were similar enough to make damage difficult to diagnose at that temperature. According to part (b) from Figure 7, the compensation technique made the results significantly easier to discern. The shifts in the signatures are eliminated leaving peaks perfectly aligned with the baseline result. Also, the CCDM indices at $35^{\circ} \mathrm{C}$ were very low and it is conceivable that smaller damage could be detected as long as the metric index threshold was accordingly set. Results across the entire temperature range were acceptable, even at the upper end of the range.
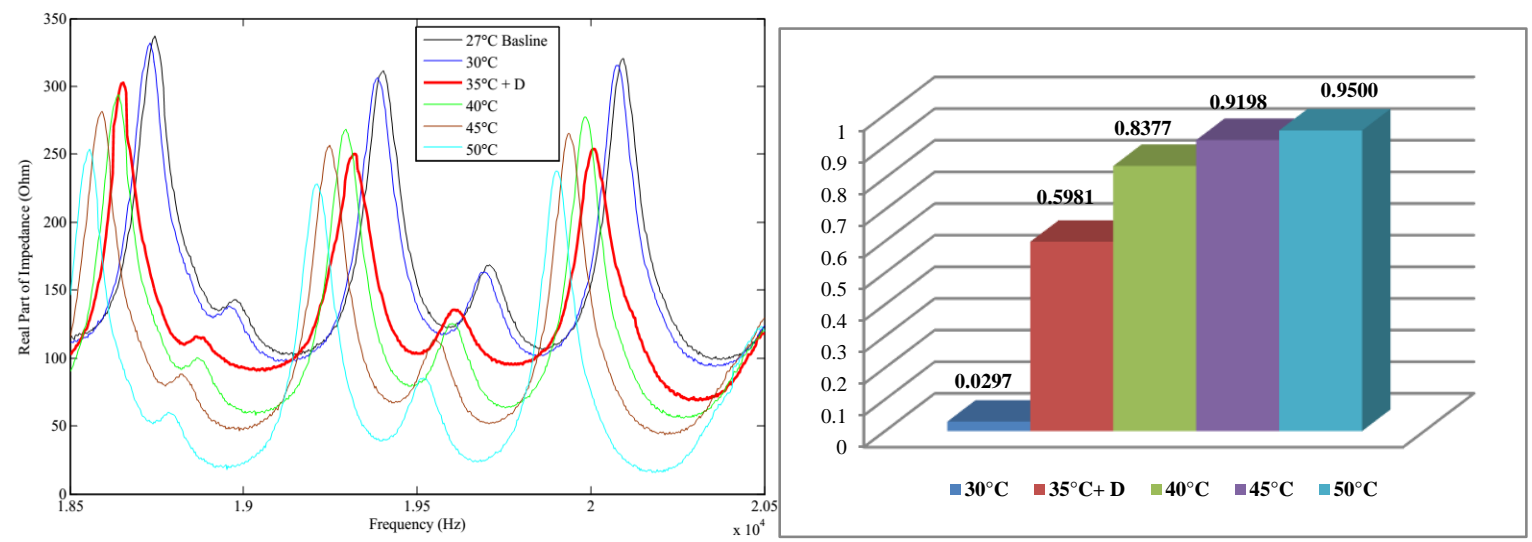

(a) 


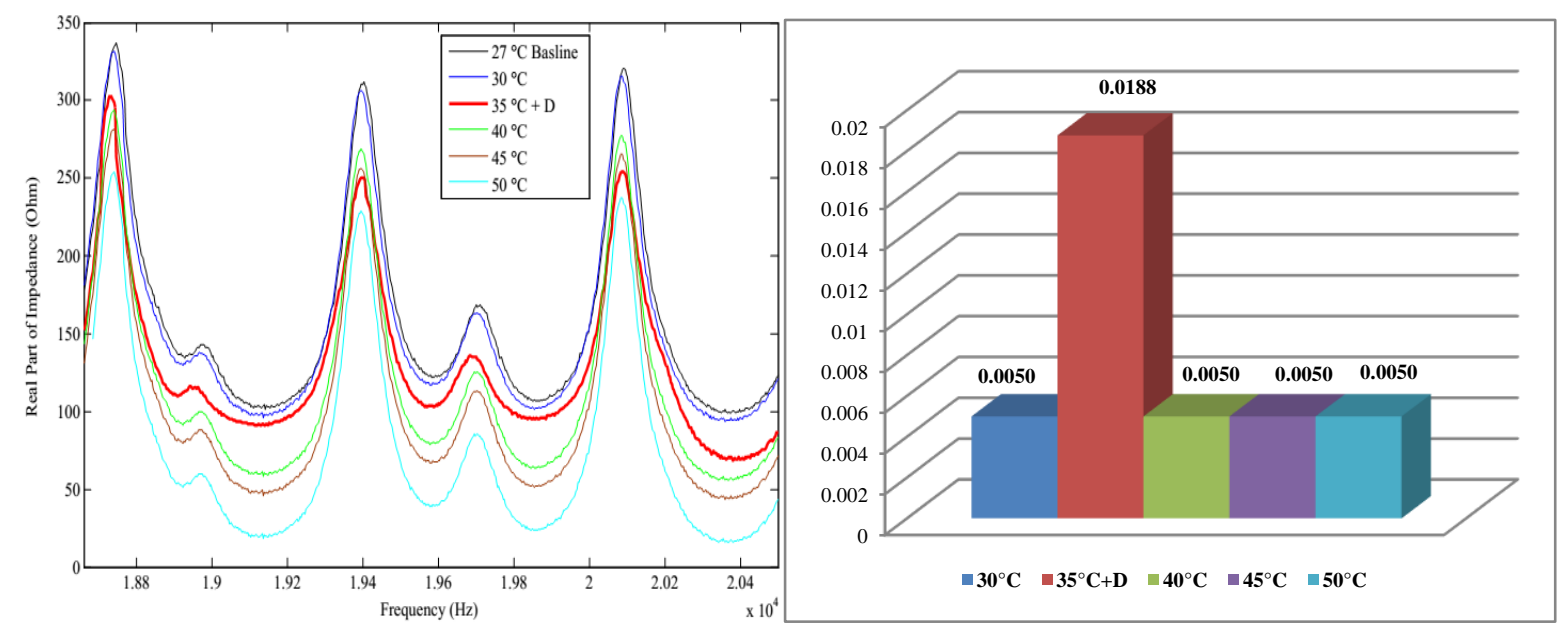

(b)

Fig. 7. FE results for Real part of impedance signatures and CCDM indices obtained for the beam (a) damage structure at $35^{\circ} \mathrm{C}$ without compensation and (b) with the proposed compensation method for the temperature effects

\section{Conclusions}

In experimental model, electrical impedance signatures of a conventional PZT sensor used in SHM were investigated along with their susceptibility to temperature variation. An aluminium specimen was also tested across a temperature range. As anticipated, the PZT dynamic response changed significantly with temperature variation. The change in the impedance signature was significant enough to cause false positive results, detect the damage that had not occurred and locate the structure's integrity into question because of temperature change. Accordingly, a temperature compensation technique was proposed to measure the exact effect of temperature change and to eliminate results ambiguity due to that temperature variation. This is considered essential to realtime health monitoring of structures in real-world conditions. Temperature effects remain critical issues in EMI structural health monitoring. This is even more obvious when it comes to detecting nascent or small damage. Effective compensation techniques are key to continued development in this field.

\section{Acknowledgement}

This research was funded by a grant from International Islamic University Malaysia Research Management Grant [grant number/project id: RMCG20-032-0032].

\section{References}

[1] Giurgiutiu, Victor. "Tuned Lamb wave excitation and detection with piezoelectric wafer active sensors for structural health monitoring." Journal of Intelligent Material Systems and Structures 16, no. 4 (2005): 291-305. https://doi.org/10.1177/1045389X05050106

[2] Djemana, M., M. Hrairi, and Y. Al Jeroudi. "Using electromechanical impedance and extreme learning machine to detect and locate damage in structures." Journal of Nondestructive Evaluation 36, no. 2 (2017): 39. https://doi.org/10.1007/s10921-017-0417-5

[3] Sepehry, Naserodin, Mahnaz Shamshirsaz, and Ali Bastani. "Experimental and theoretical analysis in impedancebased structural health monitoring with varying temperature." Structural Health Monitoring 10, no. 6 (2011): 573 585. https://doi.org/10.1177/1475921710388338

[4] Baptista, Fabricio G., Jozue Vieira Filho, and Daniel J. Inman. "Real-time multi-sensors measurement system with temperature effects compensation for impedance-based structural health monitoring." Structural Health Monitoring 11, no. 2 (2012): 173-186. https://doi.org/10.1177/1475921711414234 
[5] Baptista, Fabricio G., Danilo E. Budoya, Vinicius AD De Almeida, and Jose Alfredo C. Ulson. "An experimental study on the effect of temperature on piezoelectric sensors for impedance-based structural health monitoring." Sensors 14, no. 1 (2014): 1208-1227. https://doi.org/10.3390/s140101208

[6] Safaeifar, Hossein, and Abdollah Karimi. "The effect of temperature on the natural frequency." Teknologi Tanaman 12, no. 2 (2015): 520-528.

[7] Wandowski, Tomasz, Pawel H. Malinowski, and Wieslaw M. Ostachowicz. "Temperature and damage influence on electromechanical impedance method used for carbon fibre-reinforced polymer panels." Journal of Intelligent Material Systems and Structures 28, no. 6 (2017): 782-798. https://doi.org/10.1177/1045389X16657423

[8] Gianesini, Bárbara M., Nicolás E. Cortez, Rothschild A. Antunes, and Jozue Vieira Filho. "Method for removing temperature effect in impedance-based structural health monitoring systems using polynomial regression." Structural Health Monitoring 20, no. 1 (2021): 202-218. https://doi.org/10.1177/1475921720917126

[9] Abbas, Saqlain, Fucai Li, Zulkarnain Abbas, Taufeeq Ur Rehman Abbasi, Xiaotong Tu, and Riffat Asim Pasha. "Experimental Study of Effect of Temperature Variations on the Impedance Signature of PZT Sensors for Fatigue Crack Detection." Sound \& Vibration 55, no. 1 (2021): 1-18. https://doi.org/10.32604/sv.2021.013754

[10] Djemana, M., and M. Hrairi. "Modelling and simulation of impedance-based damage monitoring of structures." International Journal Simulation Modeling 15, no. 3 (2016): 395-408. https://doi.org/10.2507/IJSIMM15(3)1.338

[11] Lim, Hyung Jin, Min Koo Kim, Hoon Sohn, and Chan Yik Park. "Impedance based damage detection under varying temperature and loading conditions." NDT \& E International 44, no. 8 (2011): 740-750. https://doi.org/10.1016/i.ndteint.2011.08.003

[12] Bastani, Ali, Hamidreza Amindavar, Mahnaz Shamshirsaz, and Naserodin Sepehry. "Identification of temperature variation and vibration disturbance in impedance-based structural health monitoring using piezoelectric sensor array method." Structural Health Monitoring 11, no. $3 \quad$ (2012): 305-314. https://doi.org/10.1177/1475921711427486

[13] Xu, Dongyu, Sourav Banerjee, Yanbing Wang, Shifeng Huang, and Xin Cheng. "Temperature and loading effects of embedded smart piezoelectric sensor for health monitoring of concrete structures." Construction and Building Materials 76 (2015): 187-193. https://doi.org/10.1016/i.conbuildmat.2014.11.067

[14] Giurgiutiu, Victor, and Craig A. Rogers. "Recent advancements in the electromechanical (E/M) impedance method for structural health monitoring and NDE." In Smart Structures and Materials 1998: Smart Structures and Integrated Systems, vol. 3329, pp. 536-547. International Society for Optics and Photonics, 1998. https://doi.org/10.1117/12.316923

[15] Liu, Weiping, and Victor Giurgiutiu. "Finite element simulation of piezoelectric wafer active sensors for structural health monitoring with coupled-filed elements." In Sensors and Smart Structures Technologies for Civil, Mechanical, and Aerospace Systems 2007, vol. 6529, p. 65293R. International Society for Optics and Photonics, 2007. https://doi.org/10.1117/12.715238

[16] de Almeida, Vinicius Augusto Daré, Fabricio Guimarães Baptista, and Paulo Roberto de Aguiar. "Piezoelectric transducers assessed by the pencil lead break for impedance-based structural health monitoring." IEEE Sensors Journal 15, no. 2 (2014): 693-702. https://doi.org/10.1109/JSEN.2014.2352171

[17] Kee, Yoong Yen, Yutaka Asako, Tan Lit Ken, and Nor Azwadi Che Sidik. "Uncertainty of Temperature measured by Thermocouple." Journal of Advanced Research in Fluid Mechanics and Thermal Sciences 68, no. 1 (2020): 54-62. https://doi.org/10.37934/arfmts.68.1.5462

[18] Basheerali, Arafathali Shaikdawood, Meftah Hrairi, and Jaffar Syed Mohamed Ali. "Finite Element Analysis of Thermal Stress Intensity Factors for Cracked Bimaterial System Under Convective Cooling." CFD Letters 10, no. 2 (2018): 18-27.

[19] $\mathrm{Xu}$, Guidong, Baiqiang $\mathrm{Xu}$, Chenguang $\mathrm{Xu}$, and Ying Luo. "The effect of damage and temperature on electrical impedance of the PZT-beam coupled structure." Journal of Vibroengineering 18, no. 6 (2016): 3557-3567. https://doi.org/10.21595/jve.2016.16767

[20] Park, Gyuhae, Kazuhisa Kabeya, Harley H. Cudney, and Daniel J. Inman. "Impedance-based structural health monitoring for temperature varying applications." JSME International Journal Series A Solid Mechanics and Material Engineering 42, no. 2 (1999): 249-258. https://doi.org/10.1299/ismea.42.249

[21] Koo, Ki-Young, Seunghee Park, Jong-Jae Lee, and Chung-Bang Yun. "Automated impedance-based structural health monitoring incorporating effective frequency shift for compensating temperature effects." Journal of Intelligent Material Systems and Structures 20, no. 4 (2009): 367-377. https://doi.org/10.1177/1045389X08088664

[22] Sun, Fanping P., Zaffir A. Chaudhry, Craig A. Rogers, M. Majmundar, and Chen Liang. "Automated real-time structure health monitoring via signature pattern recognition." In Smart Structures and Materials 1995: Smart Structures and Integrated Systems, vol. 2443, pp. 236-247. International Society for Optics and Photonics, 1995. https://doi.org/10.1117/12.208261 